\title{
Contagious equine metritis in Portugal: A retrospective report of the first outbreak in the country and recent contagious equine metritis test results
}

\author{
T. Rocha* \\ Bacteriology Laboratory, National Reference Laboratory for CEM, Instituto Nacional de Investigação Agrária e \\ Veterinária- INIAV (National Institute of Agrarian and Veterinary Research), Avenida da República, Quinta do \\ Marquês, 2784-157 Oeiras, Portugal
}

\begin{abstract}
Contagious equine metritis (CEM), a highly contagious bacterial venereal infection of equids, caused by Taylorella equigenitalis, is of major international concern, causing short-term infertility in mares. Portugal has a long tradition of horse breeding and exportation and until recently was considered CEM-free. However, in 2008, T. equigenitalis was isolated at our laboratory from a recently imported stallion and 2 mares from the same stud. Following this first reported outbreak, the Portuguese Veterinary Authority (DGVA) performed mandatory testing on all remaining equines at the stud $(\mathrm{n}=30)$, resulting in a further 4 positive animals. All positive animals were treated and subsequently tested negative for T. equigenitalis. Since this outbreak, over 2000 genital swabs from Portuguese horses have been tested at our laboratory, with no further positive animals identified. The available data suggests that this CEM outbreak was an isolated event and we have no further evidence of CEM cases in Portugal, however, an extended and wider epidemiological study would be needed to better evaluate the incidence of the disease in Portuguese horses.

Keywords: Contagious disease, Contagious equine metritis, Equine, Reproduction, Taylorella equigenitalis.
\end{abstract}

\section{Introduction}

Contagious equine metritis (CEM), a highly contagious venereal disease of horses, is of major international concern, with important repercussions for the horse industry. It was first reported as a previously undescribed disease of horses in the United Kingdom and Ireland (Crowhurst, 1977; Platt et al., 1977), where it ravaged the Thoroughbreds racing industry in the late 70 's and early 80 's. Since then, the disease has been recorded in various horse breeds world-wide (Timoney, 1996; Ozgur et al., 2001). The causative agent of CEM was first described as Haemophilus equigenitalis (Taylor et al., 1978) and later classified into a new genus as Taylorella equigenitalis (Sugimoto et al., 1983). Two biotypes have been identified, either sensitive or resistant to streptomycin (Platt and Taylor, 1982). The acute infection in mares can cause endometritis, cervicitis or vaginitis of variable severity (with a mucopurulent vaginal discharge ranging from minimal to copious), that usually result in temporary infertility and, rarely, in abortion; clinical recovery occurs in the majority of cases, but some mares may become carriers and foals infected at foaling can become long-term, sub-clinical carriers (Eaglesome and Garcia, 1979; Timoney and Powell, 1982; Timoney, 1996). Many primary cases of infection with T. equigenitalis in the mare are subclinical and a frequent indicator of infection is a mare returning to oestrus prematurely after being bred to a putative carrier stallion (OIE, 2012). Infected stallions are not clinically affected and act as asymptomatic carriers for months or years if not treated, transmitting the disease to mares by sexual contact or artificial insemination. Transmission can also occur through inadvertent contamination of fomites during handling of the equines at time of breeding (Timoney, 1996; Schulman et al., 2013). The isolation and identification of Taylorella equigenitalis is the OIE (World Organization for Animal Health) approved method for diagnosis and the prescribed method for the international trade (OIE, 2012).

Portugal has a long tradition of horse breeding and exportation to many countries, and there is an increasing interest in the Lusitano horse, both within the country and internationally. Until recently, Portugal was considered CEM-free, however, in May 2008, T. equigenitalis was isolated for the first time (Rocha, 2014). This communication reports on the laboratory findings of this outbreak and subsequent follow up, as well as routine CEM testing data at the Instituto Nacional de Investigação Agrária e Veterinária (INIAV) for the following years, up to the present date.

\section{Animals tested}

\section{Materials and Methods}

Following the detection of the first positive case of $T$. equigenitalis in Portugal, from an imported stallion, in May 2008 (Rocha, 2014) and from 2 mares at the same stud in June, mandatory testing of all remaining equines

*Corresponding Author: Teresa Rocha. Instituto Nacional de Investigação Agrária e Veterinária- INIAV, Avenida da 
on the premises was performed by the Portuguese veterinary authority in Portugal (Direcção Geral de Alimentação e Veterinária - DGAV), consisting of 30 animals, 8 stallions and 22 mares. In addition to the animals involved in the outbreak, a total of 2070 swabs from apparently healthy Portuguese equines; 437 (21.1\%) from mares and 1633 (78.9\%) from stallions, were tested in our laboratory between May 2008 and the end of 2015. These swabs were taken from horses due for export or prior to breeding or semen collection and exportation. The 2070 samples corresponded to a total of unique 736 animals (413 stallions and 323 mares). The geographical distribution of those 736 horses can be seen in Figure 1. Most of the horses originated from the main horse breeding regions in continental Portugal: Lisboa e Setúbal (43.3\%), Estremadura e Ribatejo (26.8\%) and Alentejo (17.4\%).

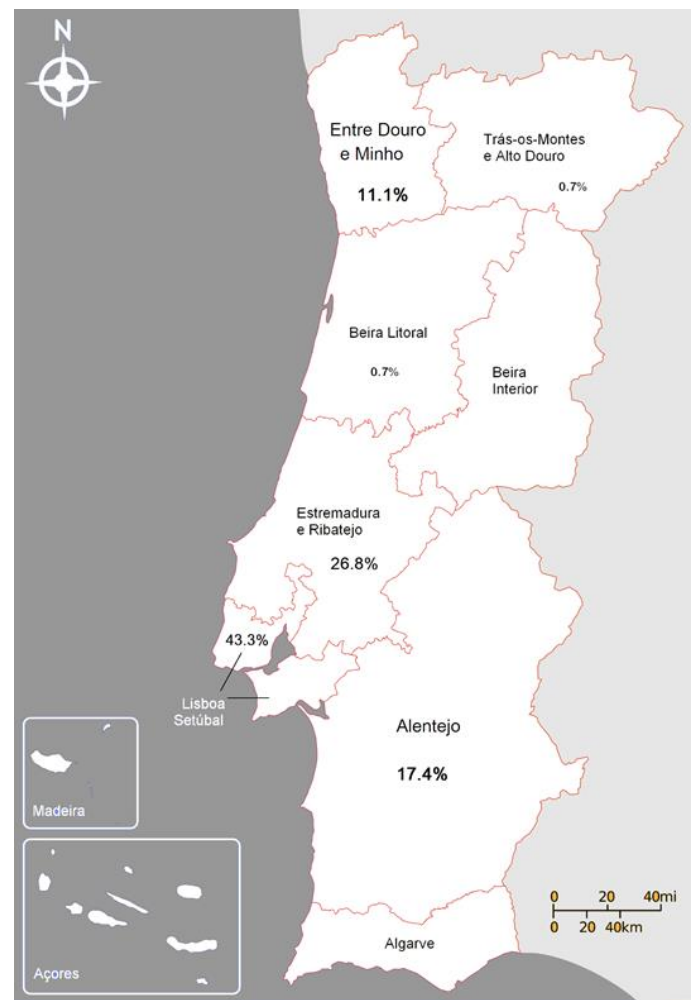

Fig. 1. Geographical distribution of the 736 horses (2070 samples) submitted for routine CEM testing at the INIAV between 2008 and 2015.

In what concerns the breeds of the tested animals, all outbreak animals were from the Lusitano breed; From the 736 horses further tested for CEM screening, only 326 of them had mention to the race on the files submitted to our laboratory and $71 \%$ of those were the Lusitano breed, while the remaining belonged to several breeds, like Arabian, Selle Francais, Holsteiner, Hanoverian, Portuguese Cruzado, Portuguese-sporthorse, Pure Spanish Race and Dutch KWPN.

\section{Quality control at our laboratory}

T. equigenitalis is very difficult to isolate owing to its fastidious nature and the concomitant presence of commensal or other bacteria and possibly fungi in the genital tract of the horse. INIAV is the national reference laboratory for CEM in Portugal and since 2006 has participated in a Quality Assessment Scheme organized by the OIE reference laboratory for CEM (VLA, Bury St. Edmunds, UK),

Sampling and transport of samples to the laboratory

During the outbreak investigation, swabs were taken from the clitoral fossa and uterus in mares (2 swabs/anatomical site/mare) and from in stallions 2 swabs were taken, one from the urethra and urethral fossa (fossa glandis) and another from the penis and penile sheath (prepuce). The swabbing protocols in screening for CEM diagnosis and recommended anatomical sites have been described previously and these procedures are a part of the veterinary practice in handling equines (Watson, 1997; HBLB, 2004). The 2070 samples for routine testing were, in mares, from the clitoris/clitoral fossa and, in stallions from the above mentioned anatomical sites as well as semen. However, occasionally only preputial/penis samples were submitted from the stallions.

All swabs were submerged in Amies charcoal transport medium and transported under cool conditions to the laboratory to be cultured within 48 hours of collection (HBLB, 2004; OIE, 2012). Only swabs under those conditions are accepted for CEM testing at the INIAV. The sterile swabs in Amies charcoal transport medium for sampling are available commercially, so they can be purchased by the veterinaries, but most of them were collected from our laboratory, since they are always granted by the INIAV when requested by the veterinaries as part of each of the analysis to be done at the INIAV.

\section{Bacteriological isolation methods and identification of positives}

Culture media were prepared at the INIAV and both the culturing and the identification of $T$. equigenitalis isolates were performed according to recommended procedures (OIE, 2012), including latex agglutination (Monotayl, Bionor Laboratories, Skien, Norway). Three different "chocolate" blood agar media were used, one with no inhibitors (inhibitors may prevent the isolation of some strains of $T$. equigenitalis), one containing streptomycin sulphate $(200 \mu \mathrm{g} / \mathrm{ml})$ to allow for the differentiation of Streptomycin-sensitive or resistant biotypes, and the third medium according to Timoney et al. (1982), a medium with inhibitors, containing $1 \mu \mathrm{g} / \mathrm{ml}$ trimethoprim, $5 \mu \mathrm{g} / \mathrm{ml}$ clindamycin and $5 \mu \mathrm{g} / \mathrm{ml}$ amphotericin B, which allows the growth of both streptomycin resistant and sensitive biotypes and is particularly useful to suppress growth of commensal bacteria and inhibit fungal growth (OIE, 2012). 
Plates were incubated at $37^{\circ} \mathrm{C}$ in a $5 \%(\mathrm{v} / \mathrm{v}) \mathrm{CO}_{2}$ incubator and an incubation period of 10 days with no growth of suspect colonies was allowed before considering specimens negative for $T$. equigenitalis.

The first $\mathrm{T}$. equigenitalis strain isolated in Portugal (strain 18456) (Rocha, 2014) was sent for confirmation to the OIE reference laboratory for CEM (VLA, Bury St. Edmunds, UK), which was done both by observation of the phenotypic characteristics and by real-time PCR (Wakeley et al., 2006). All other 6 isolates obtained during the outbreak investigation, from the first 2 positive mares (M1 and M2) and the next 3 positive mares and 1 stallion (M3, M4, M5 and St2), besides the phenotypic and monoclonal antibodies identification as T. equigenitallis, were further tested at the INIAV according to the method described by Anzai et al. (1999), a single step PCR test in a $2 \%$ agarose gel, using the primer set P1-N2, which amplifies a 445-bp DNA fragment of $T$. equigenitallis, allowing for its specific detection (Fig. 2).

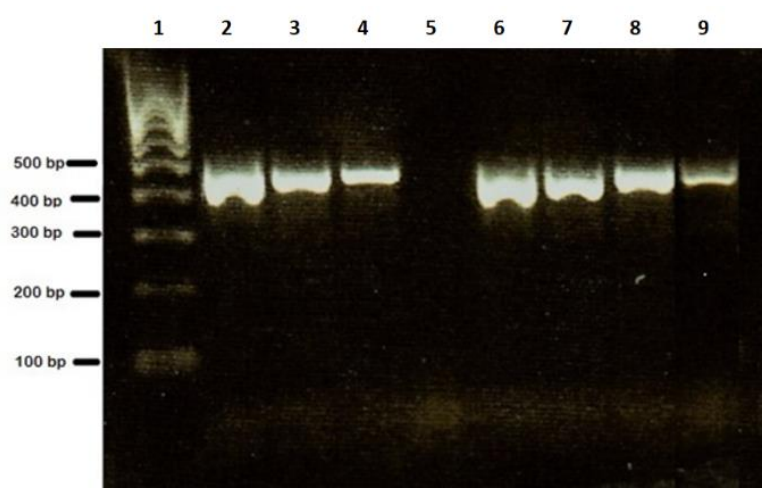

Fig. 2. PCR amplification of total DNAs of T. equigenitalis field isolates obtained from the CEM outbreak by single-step PCR with primer set P1-N2 in $2 \%$ agarose gel. Lane 1: Molecular weight marker HyperLadder IV-100bp; Lane 2: M1; Lane 3: M2; Lane 4: St 2; Lane 5: Negative control (water); Lane 6: M3; Lane 7: M4; Lane 8: M5; Lane 9: Positive control ( $T$. equigenitalis strain from Veterinary Laboratories Agency-VLA, Bury St. Edmunds, UK).

\section{Treatment of positive animals and collection of samples after treatment}

Stallions and mares were treated by thoroughly cleaning the extended penis (stallions) or the clitoral area (mares) with chlorhexidine surgical scrub and then applying nitrofurazone ointment. The treatment is done daily for 5 days, and the animals retested 8-10 days after treatment. Following treatment, 3 sets of genital swabs from all positive horses, taken approximately 10 days apart, were submitted for the bacteriological testing for T. equigenitalis.

All treatment and swab collection was undertaken by a competent veterinary surgeon designated by the DGVA.

\section{Results}

The identity of the first the first $T$. equigenitalis isolation in Portugal (strain 18456, from a recently imported stallion) was confirmed by the OIE reference laboratory for CEM (VLA, Bury St. Edmunds, UK). In addition to 2 positive mares identified in June 2008, mandatory testing of all remaining equines at the stud $(n=30)$ resulted in the isolation of T. equigenitalis from a further 4 animals, 3 mares and 1 stallion. All strains were sensitive to streptomycin.

Following treatment, all T. equigenitalis-positive equines tested negative on 3 serial cultures of genital swabs taken 10 days apart.

Since May 2008 to date, all bacteriological cultures performed at our laboratory of 2070 genital swabs from stallions and mares from various Portuguese stud farms, have yielded negative results for $T$. equigenitalis.

\section{Discussion}

Since CEM was first recognized as an emergent disease of equids in 1977, the causal agent, T. equigenitalis, has attained worldwide distribution owing to the shipment of carrier stallions and mares both within and between countries and sporadic epizootics have been confirmed in equine populations in over 29 countries in Europe and North and South America, as well as in Japan and Australia (Timoney, 2011; Schulman et al., 2013). Horse importing countries outside Europe, like the USA, have considered all countries of the European Union, and a small number of other European countries outside the EU, to be affected with this disease (USDAAPHIS, 2014), despite that information on its occurrence in Europe is scarce.

International transparency regarding the presence of CEM in a country, as well as on the actions undertaken to prevent and control its spread would be very beneficial to individual horse breeders and to the international equine trade as a whole and will ultimately assist in preventing the spread of the disease and improving the general health status of equines worldwide.

Portugal has a long tradition of horse breeding and exportation to many countries, (including the UK, Brazil, Colombia and the U.S.A.). There is increasing interest in the Lusitano horse, both within the country and internationally, and there is also an increased demand for assisted reproductive techniques such as artificial insemination in the horse industry. The control of venereally-transmitted diseases is crucial to any breeding program or for successful export, and genital swabs are regularly collected from Portuguese horses and submitted to our laboratory for $T$. equigenitalis screening.

The isolation of T. equigenitalis in Portugal for the first time in 2008 produced major concern in the equine industry. T. equigenitalis was first isolated from a 
recently imported stallion from Germany, which entered Portugal with a negative certificate for $T$. equigenitalis. The exact origin of this infection could not be confirmed with the information available to the INIAV. Although 2 positive mares were detected on the same premises shortly after the stallion, it could not be confirmed whether the stallion was used for breeding prior to testing at our laboratory, thereby acquiring $T$. equigenitalis from those mares, or if the stallion was a carrier of T. equigenitalis despite his negative CEM certificate and had in fact transmitted the infection to the mares.

Outbreak investigation confirmed a further $4 T$. equigenitalis-positive animals and following treatment and repeat serial negative post-treatment testing, the outbreak was considered controlled by the DGVA.

The isolation of $T$. equigenitalis from a stallion believed to be negative for $T$. equigenitalis reinforces the insidious nature of this disease and reiterates the fact that effective prevention and control of CEM must include a comprehensive testing program to allow for early detection and treatment of carriers animals, particularly stallions.

This paper reports the first occurrence of $T$. equigenitalis in Portugal, a country previously considered free of CEM. This outbreak was limited to one stud, where all positive animals were subsequently treated and tested negative on following up testing. These facts and the subsequent negative testing of over 2000 equine samples from 2008 to present at our laboratory, suggest that this was an isolated outbreak. However, a more extensive monitoring would be useful and required to monitor the incidence of CEM and identify possible further incursions of the disease into Portugal, given the insidious nature of the disease, the international trade in horses across borders and the constant presence of $T$. equigenitalis in neighboring European countries (Fig. 3) creating ideal conditions for spread of the disease.

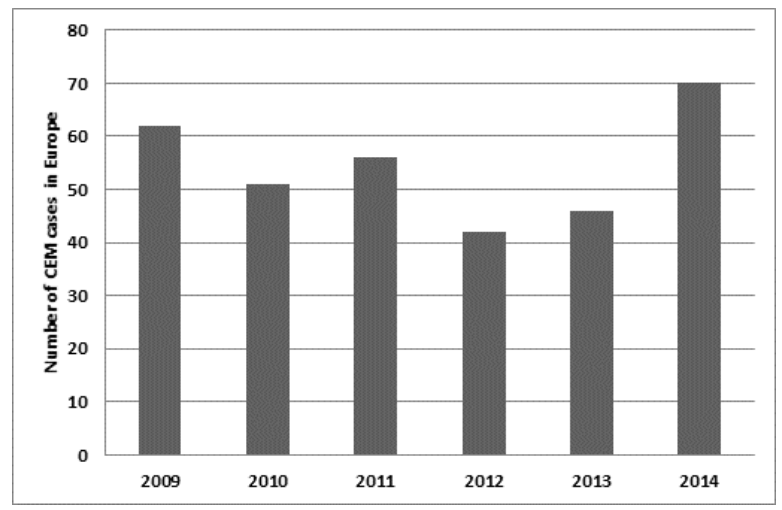

Fig. 3. Number of CEM cases in Europe, 2009-2014. (Source: EU Reference Laboratory for equine diseases, Anses, France).

\section{Conflict of interest:}

The author declares that there is no conflict of interest. Acknowledgments

The author wishes to thank Conceição Baptista and Ana Vaz for the preparation of media for T. equigenitalis isolation; Mr. Paul Todd, Work Group Leader and OIE Test consultant for CEMO (VLA, Bury St. Edmunds) for technical advice and both Mr. Paul Todd and the VLA team for the confirmation of the identification of strain 18456, the first $T$. equigenitalis isolate in Portugal; Lurdes Clemente for technical help in further testing the T. equigenitalis isolates by PCR; Sandrine Petry, from the EU Reference Laboratory for Equine Diseases for the granting of data on number of cases of CEM in European countries.

\section{References}

Anzai, T., Eguchi, M., Sekizaki, T., Kamada, M., Yamamoto, K. and Okuda, T. 1999. Development of a PCR test for rapid diagnosis of contagious equine metritis. J. Vet. Med. Sci. 61, 1287-1292.

Crowhurst, R.C. 1977. Genital infection in mares. Vet. Rec. 100(22), 476.

Eaglesome, M.D. and Garcia, M.M. 1979. Contagious Equine Metritis: A Review. Can. Vet. J. 20, 201206.

HBLB. 2004. HORSERACE BETTING LEVY BOARD Codes of Practice on Contagious equine metritis (CEM), Equine viral arteritis (EVA), Equine herpesvirus (EHV), Guidelines on strangles. Edited by: L. Archer and J.F. Wade.

OIE. 2012. Chapter 2.5.2 : Contagious Equine Metritis. OIE Terrestrial Manual. http://www.oie.int/fileadmin/Home/fr/Health_stan dards/tahm/2.05.02_CEM.pdf.

Ozgur, N.Y., Ikiz, S., Carioglu, B., Kilicarslan, R., Yilmaz, H., Akay, O. and Ligaz, A. 2001. Contagious equine metritis in Turkey: first isolation of Taylorella equigenitalis from mares. Vet. Rec. 149, 120-122.

Platt, H. and Taylor, C.E.D. 1982. Contagious Equine Metritis. In: Med. Microbiol., Vol. I. Eds: Easmon, C.S.F. and Jeljaszewicz, J., Academic Press, London, pp: 49-96.

Platt, H., Atherton, J.G., Simpson, D.J., Taylor, C.E.D., Rosenthal, R.O., Brown, D.F.J. and Wreghitt, T.G. 1977. Genital infection in mares. Vet. Rec. 101, 20.

Rocha, T. 2014. Metrite Contagiosa Equina em Portugal: Diagnóstico Laboratorial, primeiros casos positivos e dados subsequentes. In the Proceedings of the VIth Congress of the Portuguese Society of Veterinary Medicine, INIAV Oeiras, Portugal, 3-5 April 2014, pp: 233.

Schulman, M.L., May, C.E., Keys, B. and Guthrie, A.J. 2013. Contagious equine metritis: Artificial 
reproduction changes the epidemiologic paradigm. Vet. Microbiol. 167(1-2), 2-8.

Sugimoto, C., Isayama, Y., Sakazaki, R. and Kuramochi, S. 1983. Transfer of Haemophilus equigenitalis Taylor et al., 1978 to the genus Taylorella gen. nov. as Taylorella equigenitalis comb.-nov. Curr. Microbiol. 9, 155-162.

Taylor, C.E., Rosenthal, R.O., Brown, D.F.J., Lapage, S.P., Hill, L.R. and Legros, R.M. 1978. The causative organism of contagious equine metritis 1977: proposal for a new species to be known as Haemophilus equigenitalis. Equine Vet. J. 10(3), 136-144.

Timoney, P.J. 1996. Contagious equine metritis. Comp. Immunol. Microbiol. Infec. Dis. 19(3), 199-204.

Timoney, P.J. 2011. Horse Species Symposium: Contagious Equine Metritis: An insidious threat to the horse breeding industry in the United States. J. Anim. Sci. 89(5), 1552-1560.
Timoney, P.J. and Powell, D.G. 1982. Isolation of the contagious equine metritis organism from colts and fillies in the United Kingdom and Ireland. Vet. Rec. 111, 478-482.

Timoney, P.J., Shin, S.J. and Jacobson, R.H. 1982. Improved selective medium for isolation of the contagious equine metritis organism. Vet. Rec. 111, 107-108.

USDA-APHIS. 2014. United States Department of Agriculture - Animal and Plant Health Inspection Service (USDA-APHIS) Factsheet, May 2014.

Wakeley, P.R., Errington, J., Hannon, S., Roest, H.I.J., Carson, T., Hunt, B., Sawyer, J. and Heath, P. 2006. Development of a real-time PCR for the detection of Taylorella equigenitalis directly from genital swabs and discrimination from Taylorella asinigenitalis. Vet. Microbiol. 118, 247-254.

Watson, E. 1997. Swabbing protocols in screening for contagious equine metritis. Vet. Rec. 140, 268-271. 